\title{
Experimental verification of microbending theory using mode coupling to discrete cladding modes
}

Probst, C. B.; Bjarklev, Anders Overgaard; Andreasen, S. B.

Published in:

Journal of Lightwave Technology

Link to article, DOI:

$10.1109 / 50.17733$

Publication date:

1989

Document Version

Publisher's PDF, also known as Version of record

Link back to DTU Orbit

Citation (APA):

Probst, C. B., Bjarklev, A. O., \& Andreasen, S. B. (1989). Experimental verification of microbending theory using mode coupling to discrete cladding modes. Journal of Lightwave Technology, 7(1), 55-61.

https://doi.org/10.1109/50.17733

\section{General rights}

Copyright and moral rights for the publications made accessible in the public portal are retained by the authors and/or other copyright owners and it is a condition of accessing publications that users recognise and abide by the legal requirements associated with these rights.

- Users may download and print one copy of any publication from the public portal for the purpose of private study or research.

- You may not further distribute the material or use it for any profit-making activity or commercial gain

- You may freely distribute the URL identifying the publication in the public portal 


\title{
Experimental Verification of Microbending Theory Using Mode Coupling to Discrete Cladding Modes
}

\author{
C. B. PROBST, A. BJARKLEV, AND S. B. ANDREASEN
}

\begin{abstract}
In this paper the existence of discrete cladding modes in single mode fibers is illustrated. This is done by inducing periodically repeated microbends along the fiber axis, whereupon spectral measurements of the additional loss are performed. In order to explain the results of these measurements, it is necessary to apply a microbending theory in which coupling between the guided mode and a number of discrete cladding modes is considered. Very good agreement between theory and measurements is achieved. We will finally discuss the consequences of the existence of discrete cladding modes on the proper choice of artificial microbending spectrum used for fiber characterization.
\end{abstract}

\section{INTRODUCTION}

OHARACTERIZATION of single mode fibers often includes measurements of microbending sensitivity. Well known methods to measure the additional microbending loss are the basket weave test [1], where the fiber is wound with different tensions in multiple levels on a spool, and the sandpaper test, which makes use of the deformations that appear when the fiber is pressured against sandpaper [2]. It has however been argued [3] that one of the problems of using the existing tests is that the deformations are difficult to reproduce. It has therefore been proposed that one should use a deformation device with a well defined spatial deformation spectrum, and attention has been drawn towards standard sieves [3] or equally spaced pins.

In the interpretation of the different microbending test results a number of calculation methods have been applied. One approach is to derive the loss coefficient from a coupling between the guided mode in the fiber and a continuous spectrum of lossy cladding modes [4]-[6]. In another approach a summation is made of the coupling contributions due to coupling between the fundamental mode field and discrete cladding modes [7], [8].

It is however important to notice that these calculation methods all are developed considering a broad spectrum of spatial frequencies describing the deformations, and they will, for a large range of such spectra, show almost

Manuscript received September 2, 1987. This work was supported by Telecom Denmark.

C. B. Probst and A. Bjarklev are with the Electromagnetics Institute, Technical University of Denmark, DK-2800 Lyngby, Denmark.

S. B Andreasen was with the Electromagnetics Institute, Technical University of Denmark, DK-2800 Lyngby, Denmark. He is now with the R\&D Laboratory, Jutland Telephone, DK-8310 Tranbjerg J., Denmark. IEEE Log Number 8821125 . identical results. If we on the other hand use reproducible microbending spectra such as those made by standard sieves, it becomes possible to make certain spatial frequencies dominant simply by periodic repetition of the deformation acting on the fiber. The results from different calculation methods will in these situations show considerable deviations, and it is therefore necessary to derive more information about the actual nature of the microbending losses in order to choose a proper theoretical model for the special test situations.

The purpose of this paper is therefore to show that it is possible to illustrate the existence of discrete cladding modes from a simple spectral microbending loss measurement, as long as a periodically repeated deformation along the fiber axis is used.

In Section II the microbending loss coefficient is evaluated considering power coupling between the guided mode and a number of discrete cladding modes. It is furthermore shown how the deformation spectrum describing the microbends at the fiber core axis can be derived from knowledge of periodically repeated deformations at the primary fiber coating surface, and the spectrum is illustrated for the actual deformation device used in the measurements.

The spectral loss measurements made by the use of a standard attenuation measurement setup are discussed in Section III, and comparisons are made with theoretical curves. Based on these results it is finally considered in Section IV how a proper choice of microbending deformation device can be made for the purpose of characterizing single mode fibers.

\section{THEORY}

Deformations along the longitudinal direction of a single mode fiber will cause power to be lost from the fundamental mode. The purpose of this section is to show how the spectral dependence of such losses can be calculated when the fiber is exposed to the action of a periodically repeated microbending. This is done first by resuming the perturbation theory [9]-[10] describing coupling between the guided mode and the cladding modes of a distorted single mode fiber. Secondly the deformation spectrum is calculated for the actual deformation device used in this work, and the spectral loss dependence is illustrated using a step-index profile example. 
The fibers under consideration are assumed to be circular symmetric, and the refractive-index distribution of a perfectly straight fiber is given by $n_{0}(r)$, where $r$ is the radial coordinate of a cylindrical coordinate system. The index distribution of the imperfect fiber, which also may depend on the angular coordinate $\phi$ and the axial coordinate $z$ is represented by $n(r, \phi, z)$.

The relation between the perfect and the by microbending distorted index distributions can be expressed as a first order Taylor expansion [7]:

$$
n(r, \phi, z)=n_{0}(r)+\frac{\partial n_{0}}{\partial r} \cdot f(z) \cos \phi
$$

in which $f(z)$ describes the deformation of the fiber axis.

The coupling strength is determined by coupling coefficients, which is found as integrals in the $r, \phi$-plane over products of the kind $\left[n(r, \phi, z)-n_{0}(r)\right] \cdot E_{01} \cdot E_{l p}$ [7]. Here $E_{01}$ denotes the electric field of the $\mathrm{LP}_{01}$-mode and $E_{l p}=E_{p}^{l} \cdot \cos (l \cdot \phi)$ is the electric field of the $\mathrm{LP}_{l p^{-}}$ cladding mode. Since the guided mode field is independent of $\phi$, the coupling coefficients have nonvanishing values only for cladding modes $\mathrm{LP}_{1 p}$ which have the same $\phi$-dependence as the index perturbation $[n(r, \phi, z)-$ $\left.n_{0}(r)\right]$.

The microbending power loss formula based on the coupled mode theory [9], [10] can therefore be given as

$$
2 \alpha_{m}=\sum_{p=1}^{\infty} C_{1 p}^{2} \cdot \Phi\left(\Delta \beta_{1 p}\right) .
$$

In this expression $C_{1 p}$ is the $z$-independent coupling coefficient, which describes the coupling strength between the $\mathrm{LP}_{01}$-mode and the $\mathbf{L P}_{1}$-cladding mode. The formula shows further more that the size of each loss contribution depends on the value of the deformation spectrum $\Phi($ ) at the spatial frequency $\Delta \beta_{1 p}$, which is the difference between the propagation constants of the guided mode and the actual cladding mode $\mathrm{LP}_{1 p}$.

In the case of microbends the coupling coefficients can be written as [7], [8]

$$
C_{1 p}^{2}=\frac{k^{2}}{2} \frac{\left(\int_{0}^{\infty} \frac{\partial n_{0}}{\partial r} E_{01} E_{p}^{1} r d r\right)^{2}}{\int_{0}^{\infty} E_{01}^{2} r d r \int_{0}^{\infty}\left(E_{p}^{1}\right)^{2} r d r}
$$

where $k=2 \pi / \lambda$ is the free space wavenumber.

The electrical fields $E_{01}$ and $E_{p}^{1}$ are all calculated as solutions for the scalar wave equation found by use of the multilayer approximation [11]. Here it is important to take proper care to the influence of the core when calculating the cladding mode fields [8]. (This influence is assumed negligible in [7].) Furthermore it is important to notice, that the mode fields are set to zero outside the outer fiber radius $b=62.5 \mu \mathrm{m}$. This truncation, which corresponds to a total reflection at the radius $b$, is considered a good approximation due to the large index difference at the fiber-coating boundary.

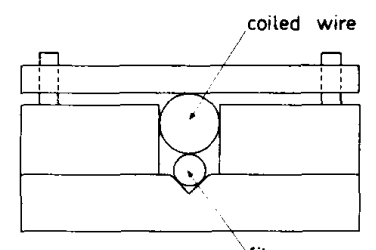

(a)

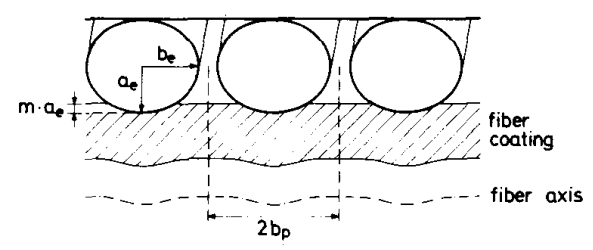

(b)

Fig. 1. (a) Cross section of the deformation device. The fiber is placed in a V-groove $24 \mathrm{~cm}$ long. The coiled wire is then placed on the fiber and pressed down with a flat load. (b) Longitudinal cross section of the coiled wire giving the surface deformation $A(z)$ on the fiber. The ellipse parameters $a_{e}$ and $b_{e}$ and the relative compression $m$ are illustrated. Also shown is the half period length $b_{p}$.

The size of the coupling coefficients $C_{1 p}$ and the propagation constant difference $\Delta \beta_{1 p}$ will, in addition to the dependence of the index profile, also change as the wavelength varies. If the deformation spectrum $\Phi(\Delta \beta)$ is therefore made to have peak values at known spatial frequencies, high loss contributions will appear at wavelengths where the peaks coincide with the position of the coupling coefficients.

In order to find such a deformation, we will turn to the calculation of the spatial power spectrum $\Phi()$, which is given from the deformation $f(z)$ of the fiber axis as [10]:

$$
\Phi(\Omega)=\frac{1}{2 L}\left|\int_{-L}^{L} f(z) \cdot \exp (-j \Omega z) d z\right|^{2} .
$$

$2 L$ is the total length of the deformations. Peaks will appear in the Fourier spectrum of (4) at well defined spatial frequencies, if the repetition period of identical fiber deformations can be precisely controlled. To make the deformation periods as uniform as possible and at the same time get a long distance over which the fiber feels the deformation, a thin homogeneous metal wire was wound closely around a center wire. We hereby get a surface as shown on Fig. 1, where it is possible to pressure the fiber against several hundred periods in order to achieve a very narrow peak on the deformation spectrum. It must be noted that no deformation of the metal is assumed, when it is pressed against the fiber coating surface.

As shown on Fig. 1, each period of the deformation at the surface of the primary coating can be represented by the elliptic curve given by the half axis $a_{e}$ and $b_{e}$ and the compression factor $m \cdot m$ is as shown in Fig. 1 defined as the percentage of the radius $a_{e}$ of the coiled wire penetrating into the coating surface. The coating surface for 
one period can be described by

$$
A_{p}(z)= \begin{cases}\frac{a_{e}}{b_{e}} \sqrt{b_{e}^{2}-z^{2}}-a_{e}(1-m), \\ 0, & \text { for }|z| \leqq b_{e} \sqrt{(2-m) m} \\ 0 & \text { otherwise. }\end{cases}
$$

The total deformation device, which includes $(2 K+1)$ periods, can then be represented by the following convolution

$$
A(z)=A_{p}(z) * \sum_{n=-K}^{K} \delta\left(z-n \cdot 2 b_{p}\right)
$$

where $\delta$ is the Dirac delta function. Note that the half period length $b_{p}$ is slightly larger than the elliptic half axis $b_{e}$, unless the wire is coiled so tight that neighboring coils on the wire touch each other. The Fourier transform of the function $A(z)$ in (6) can then be calculated as

$$
\begin{aligned}
G(\Omega)= & \int_{-\infty}^{\infty} A(z) \cdot \exp (-j \Omega z) d z \\
= & a_{e} \cdot b_{e}\left[J_{0}\left(\Omega b_{e}\right)\left(T-\frac{1}{2} \sin 2 T\right)+J_{2}\left(\Omega b_{e}\right)\right. \\
& \cdot\left(T-\frac{1}{3} \sin 2 T-\frac{1}{12} \sin 4 T\right) \\
& +\sum_{n=2}^{\infty} J_{2 n}\left(\Omega b_{e}\right)\left(\frac{\sin (2(n-1) T)}{2\left(2 n^{2}-3 n+1\right)}\right. \\
& \left.\left.+\frac{\sin (2(n+1) T)}{2\left(2 n^{2}+3 n+1\right)}-\frac{\sin (2 n T)}{n\left(4 n^{2}-1\right)}\right)\right] \\
& \cdot \frac{\sin \left((2 K+1) \Omega b_{p}\right)}{\sin \left(\Omega b_{p}\right)}
\end{aligned}
$$

where $T=\arcsin (\sqrt{(2-m) m})$ and the last factor is due to the periodicity.

From (7) it is now possible to calculate the deformation spectrum at the coating surface for given values $m, a_{e}$, and $b_{e}$. It appears however from (4) that the microbending loss coefficient is calculated from the spectrum of the core axis deformation $f(z)$. Therefore it is necessary to find a relation between the surface deformation $A(z)$ and the resulting core axis deformation. In order to do this, elasticity theory is applied to the situation illustrated in Fig. 1, where the fiber coating on one side follows the surface $A(z)$ and on the other side is mounted in a $v$-groove. The relation between $A(z)$ and $f(z)$, which describes the material compression has in [12] been expressed by a differential equation:

$$
\frac{\partial^{4}}{\partial z^{4}} f(z)+\kappa^{4} \cdot f(z)=\kappa^{4} \cdot A(z)
$$

where $\kappa$ is a constant depending on the elasticity coefficient (Young module) of the coating material.

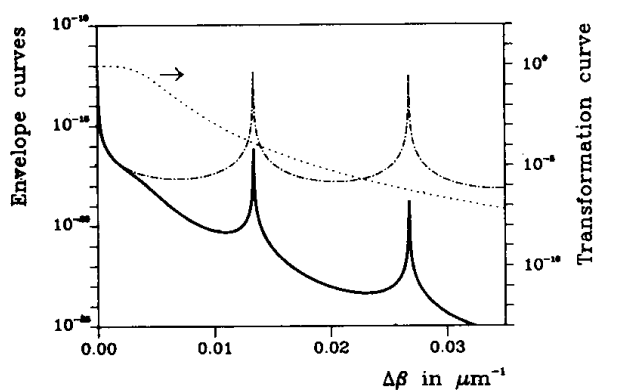

Fig. 2. Envelope curve for the deformation spectrum at the fiber coating surface as function of the spatial frequency $\Delta \beta$ (dash-dotted curve). The factor transforming the surface spectrum to the core is examplified (dotted curve) and the envelope of the final micro-deformation spectrum is shown (solid curve). The deformation device is given by the parameters $2 L=24 \mathrm{~cm}, b_{p}=235 \mu \mathrm{m}, a_{e}=212.5 \mu \mathrm{m}, b_{e}=220 \mu \mathrm{m}$, and $m$ is set to 5 percent.

By Fourier transformation of (8) we find an expression for the core axis deformation spectrum $\Phi(\Omega)$ given as

$$
\Phi(\Omega)=\frac{1}{2 L}\left[\frac{\kappa^{4}}{\kappa^{4}+\Omega^{4}}\right]^{2} G^{2}(\Omega) .
$$

In this formula $G^{2}(\Omega)$ is the power spectrum of the surface deformation function (see (7)).

As an illustration of the deformation spectrum we now look at a device made by winding a metal wire of diameter $2 a_{e}=425 \mu \mathrm{m}$ around a center wire with diameter $d=$ $500 \mu \mathrm{m}$. The elliptic curve in (5) is then given by the parameters $a_{e}=212.5 \mu \mathrm{m}$ and $b_{e}=220 \mu \mathrm{m}$. By pressuring $24 \mathrm{~cm}$ (corresponding to 511 periods) of this deformation device against a fiber using the parameter value $m=5$ percent, the surface deformation spectrum of Fig. 2 appears, and we find a number of peaks equally spaced by $\Delta \beta_{s}=1.338 \times 10^{4} \mathrm{~m}^{-1}$. The surface deformation spectrum is transformed to the core deformation spectrum by the modification described in (9). A typical example of such a modification curve is also shown on Fig. 2 together with the final microbending spectrum. The suppression of high spatial frequencies, due to the primary coating, is in this typical example seen to make the peak value at $\Delta \beta_{s}$ more than 400 times higher than the peak value at the frequency $2 \cdot \Delta \beta_{s}$. Since no coupling coefficients appear at $\Delta \beta$-values close to zero, the peak of the deformation spectrum at $\Delta \beta_{s}$ will have dominant influence on the shape of the spectral loss curve.

In order to illustrate this influence of the deformation spectrum peak value, we now look at an example where the deformation spectrum of Fig. 2 is applied on a step index fiber. The considered step index profile is given by a core radius $a=3.56 \mu \mathrm{m}$ and core cladding index difference $\Delta n=6.20 \times 10^{-3}$. This fiber has zero dispersion wavelength close to $\lambda_{0}=1.32 \mu \mathrm{m}$ and Laplace spotsize [6] $\omega_{L}\left(\lambda_{0}\right)=4.0 \mu \mathrm{m}$.

In Fig. 3 the locations in spatial frequency of the coupling coefficients are shown for three different wavelengths $(\lambda=1.405 \mu \mathrm{m}, \lambda=1.415 \mu \mathrm{m}$, and $\lambda=1.425$ $\mu \mathrm{m})$. It appears that the coupling coefficient of lowest order moves to smaller spatial frequencies $\Delta \beta_{1 p}$ as the 


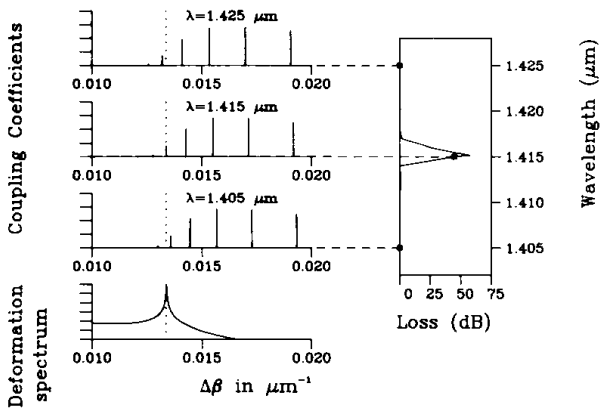

Fig. 3. Illustration of the appearance of peaks in the spectral microbending loss curve. For three different wavelengths are the coupling coefficients of a step index fiber (given by radius $a=3.56 \mu \mathrm{m}$ and index difference $\Delta n=6.20 \times 10^{-3}$ ) shown versus the propagation constant difference $\Delta \beta$. When the coupling coefficient passes the deformation spectrum peak a higher loss value is seen, which in this special case is due to coupling to the $\mathrm{LP}_{12}$-mode.

wavelength is increased. This movement of the coupling coefficients is due to the fact that the propagation constant $\beta_{01}$ of the guided mode decreases faster than the propagation constants of the lower order cladding modes, and it will result in peaks in the total microbending loss located at characteristic wavelengths. These loss peaks correspond to the situations, where the spatial frequency positions of the coupling coefficients coinsides with the peaks of the deformation spectrum.

The peaks on the spectral loss curve illustrated on Fig. 3 will not appear, if coupling to a continuous mode spectrum is considered. Also the characteristic shape of the spectral loss curve will be strongly dependent on the considered fiber design.

\section{Measurements}

In the previous section it was shown that the existence of discrete cladding modes in a single mode fiber will result in characteristic peaks in the spectral microbending loss curve, when a periodically repeated fiber deformation is applied. The purpose of this section is to show how the measured loss curves agree with the theoretical predictions and hereby illustrate the existence of discrete cladding modes.

In order to measure the spectral dependence of the microdeformation losses, a white light source and a monochromator were used. After passing a chopper, the light was launched into the fiber. Before the microbending inducing device, the fiber was coiled with a radius of 1.5 $\mathrm{cm}$ for the purpose of removing the higher order modes. The signal was after detection and amplification handled by a microcomputer, which also controlled the monochromator. The wavelength step was $\Delta \lambda=10 \mathrm{~nm}$. By this standard setup it is possible to measure the additional loss due to microbending by storing the spectral values of the transmitted power when pressuring the fiber against the deformation device and afterwards normalizing with measurements made without microdeformations.

Fibers with three different designs were measured. Two of these designs, namely step index profile fibers with and without depressed cladding, had zero dispersion wave- length close to $\lambda=1.31 \mu \mathrm{m}$. The third fiber was dispersion shifted and had a triangular core index profile.

The first measurements were made using a deformation device described by the ellipse parameters $a_{e}=212.5 \mu \mathrm{m}$ and $b_{e}=220 \mu \mathrm{m}$, and the fiber was pressured against the device over a distance of $24 \mathrm{~cm}$. The pressure was accomplished by a total weight of $7.7 \mathrm{~kg}$ and from knowledge of the elasticity constants of the coating and fiber material the relative compression $m$ and hereby the deformation spectrum were calculated.

As illustrated at the measured spectral loss curves in Fig. 4 the three different fiber designs show individually characteristic microbending sensitivity. Although the absolute values of the loss spectrum grow as the weight on the deformation device is increased, it is important to point out that for each fiber the ratios between the peak values are almost constant. The spectral positions of the peaks are fixed as the compression depth is changed and the measurement results are found to be reproducible.

The index profiles of the three different fiber designs were from preform measurements approximated by multilayer structures, and calculations were performed as outlined in the previous section. The results of these calculations are shown in Fig. 4 as solid curves.

It is obvious in each of the three cases that the calculated loss peaks appear at almost the same wavelengths as the peaks on the measured curves. We find however, that the peaks on the measured curves are broader than the calculated ones. The reason for this is partly that the light source has a spectral width of approximately $8 \mathrm{~nm}$ (half width, half maximum). We have therefore measured the losses convolved with the spectral shape of the measuring light. Another reason for the broadening of the measured spectral loss peaks is that the deformation device can not be expected to be perfectly homogeneous. The deformation spectrum will therefore have lower and broader peaks. For clarity and simplicity we have, however, chosen to neglect the broadening effects in our calculations. A detailed discussion of the absolute loss values will furthermore need a detailed description of the elasticity coefficients of the coating material and of the elasticity theory. It seems therefore more fruitful to focus at the relative mutual heights of the loss peaks and the positions on the wavelength axis.

If we in accordance to this look at the results in Fig. 4(a) for the matched clad step-index fiber, high loss peaks are found at $\lambda=1.288 \mu \mathrm{m}, \lambda=1.362 \mu \mathrm{m}$, and $\lambda=$ $1.530 \mu \mathrm{m}$. These loss peaks occur from coupling to the $\mathrm{LP}_{13^{-}}, \mathrm{LP}_{14^{-}}$, and $\mathrm{LP}_{15^{-}}$-cladding modes, respectively. As can be seen for the positions of the loss peaks, there is excellent agreement between theory and measurements. The agreement for the relative mutual hights of the peaks is somewhat poorer, though the tendency is right. The reason for this is mainly the above-mentioned broadening and lowering of the spectrum. Furthermore, one should remember that the measurements are made with discrete wavelength steps $\Delta \lambda=10 \mathrm{~nm}$. The absolute loss peak can easily lay in between two measured points. 


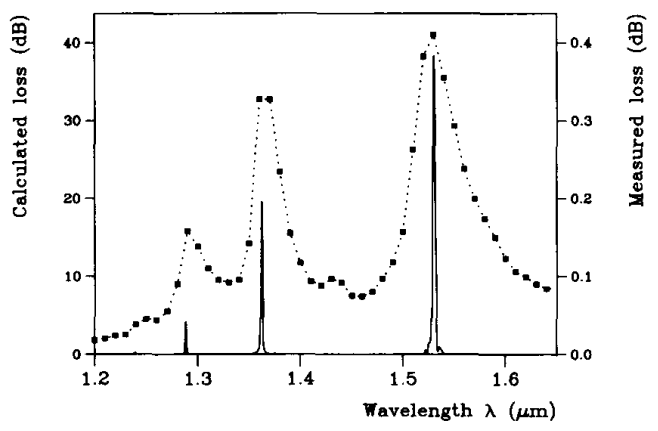

(a)

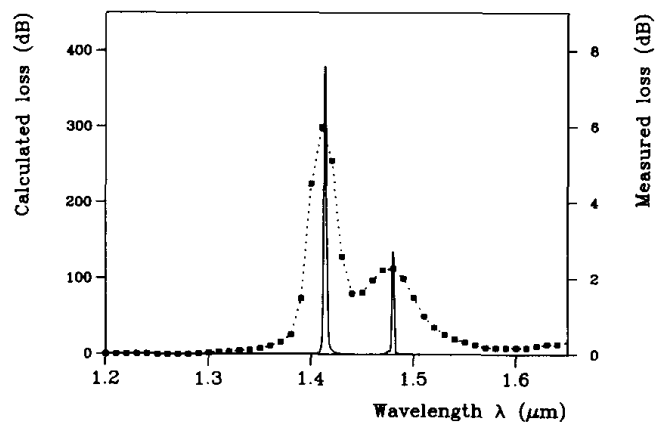

(b)

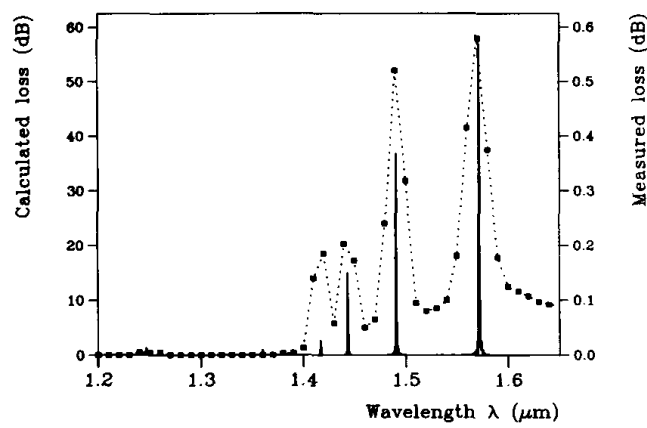

(c)

Fig. 4. Measured (dotted line and markers) and calculated (solid line) spectral microbending losses for: (a) matched cladding step index fiber, (b) depressed cladding step index fiber, (c) dispersion shifted triangular core fiber. Fiber $24 \mathrm{~cm}$ long is pressured against the deformation device with the ellipse parameters $a_{e}=212.5 \mu \mathrm{m}$ and $b_{e}=220 \mu \mathrm{m}$ and the relative compression $m=5.2$ percent. There are 511 periods.

Good agreement is also found between the calculated and measured results for the depressed clad fiber (Fig. 4(b)) and for the triangular core fiber (Fig. 4(c)). The loss peaks found using the depressed clad fiber are due to coupling to the $\mathrm{LP}_{14}$ and $\mathrm{LP}_{15}$-modes at $\lambda=1.413 \mu \mathrm{m}$ and $\lambda$ $=1.480 \mu \mathrm{m}$, respectively. The calculated loss peaks for the dispersion shifted fiber are accomplished by coupling to the $\mathrm{LP}_{11}, \mathrm{LP}_{12}, \mathrm{LP}_{13}$, and $\mathrm{LP}_{14}$-modes. The positions of these peaks are as follows: $\lambda_{11}=1.417 \mu \mathrm{m}, \lambda_{12}=$ $1.443 \mu \mathrm{m}, \lambda_{13}=1.491 \mu \mathrm{m}$, and $\lambda_{14}=1.572 \mu \mathrm{m}$. If one looks carefully at Fig. 4(c) two minor peaks on the calculated loss curve can be seen at $\lambda=1.247 \mu \mathrm{m}$ and $\lambda=$ $1.360 \mu \mathrm{m}$, respectively. These peaks can also be explained by coupling to the $\mathrm{LP}_{17}$ and $\mathrm{LP}_{18}$ modes but at the

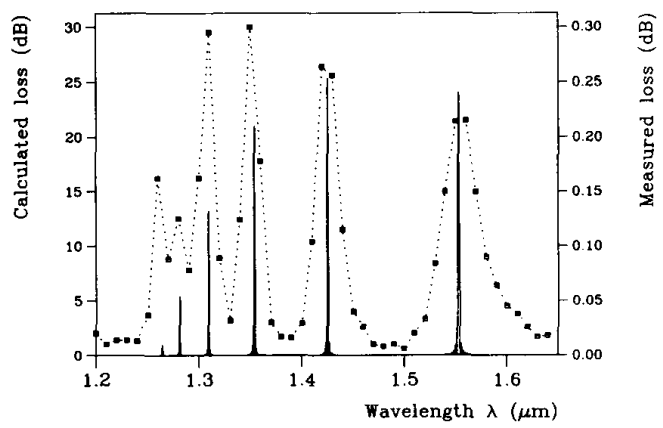

Fig. 5. Microdeformation loss shown versus the wavelength for the same dispersion shifted fiber as in Fig. 4(c). The surface deformation is now characterized by $a_{e}=160 \mu \mathrm{m}, b_{e}=165 \mu \mathrm{m}$, and $m=7.4$ percent. Fiber $24 \mathrm{~cm}$ long feels the deformation corresponding to 689 periods.

spatial frequency $2 \Delta \beta_{s}$, where the next very depressed peak of the microdeformation spectrum is located. If the scale of the measured losses is blown up, one can actually see a small bump on the curve around $\lambda=1.247 \mu \mathrm{m}$ whereas the peak at $\lambda=1.360 \mu \mathrm{m}$ is so small that no additional loss has been measured.

The choice of the deformation spectrum has a large influence on the actual shape of the spectral loss curve. This is illustrated in Fig. 5 where the additional microbending loss is measured using another deformation device, which is characterized by the parameters $a_{e}=160 \mu \mathrm{m}, b_{e}=165$ $\mu \mathrm{m}$ and a total relative compression $m=7.4$ percent. The fiber used here is the same dispersion shifted fiber as discussed above. We find that the loss peaks here can be explained by coupling to the six cladding modes $L P_{11}-$ $\mathrm{LP}_{16}$. The calculated wavelengths $\lambda_{11}-\lambda_{16}$ in micrometers are $1.265,1.281,1.309,1.354,1.425$, and 1.553, respectively.

\section{Discussion}

The actual spectral position of the loss peaks are strongly dependent on the precise shape of the index profile. In order to illustrate this sensitivity to changes in the fiber design, we now look at the step profile example used in Section II. The core radius and index difference are changed separately, and we show the spectral positions of the loss peaks due to coupling to the $\mathrm{LP}_{11^{-}}, \mathrm{LP}_{12^{-}}$, $\mathrm{LP}_{13^{-}}$, and $\mathrm{LP}_{14^{-}}$-modes on Fig. 6.

Since an increase of either the core radius $a$ or the index difference $\Delta n$ will result in better guiding of the fundamental mode, the propagation constant difference $\Delta \beta_{1 p}$ will increase. We therefore have to go to higher wavelengths before the $\Delta \beta_{1}$-values coincide with the peak on the deformation spectrum, and curves as those on Fig. 6 are found. The important thing is however that a change of no more than 1 percent on the core radius will move the $\mathrm{LP}_{11}$-mode peak to a wavelength which is $8 \mathrm{~nm}$ higher, and the $\mathrm{LP}_{14}$-mode peak is moved $11 \mathrm{~nm}$. These displacements, which are close to the spacing of the measuring points used previously, are so large that detailed knowledge of the index profile is required in order to make precise loss predictions. The mutual hights of the loss peaks 

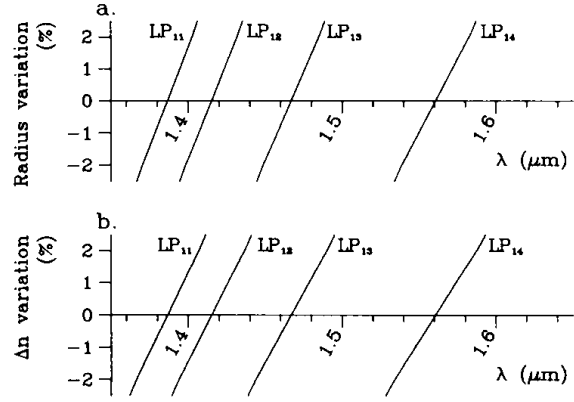

Fig. 6. Spectral positions of the microbending loss peaks shown for different values of the core radius $a$ and index difference $\Delta n$ in a step index fiber. The fiber used as point of reference is given by $a=3.56$ and $\Delta n$ $=6.20 \times 10^{-3}$

are furthermore changed, and this effect will be even clearer for more complex fiber designs.

The illustrated existence of discrete cladding modes must be taken into account, when microbending measurements for characterization of single mode fibers are planned. Namely, we have seen in Fig. 4 that if a deformation spectrum with one or a few dominant spatial frequencies are used, the added loss of one wavelength can vary orders of magnitude for different fibers even though these may have almost equal sensitivities to practical microbends. Therefore, if we only want to measure at a few wavelengths, we cannot make a fair comparison of different fibers using a periodic microbending device. Instead we must use more or less random deformations so that we get a broad continuous deformation spectrum and coupling to a large number of cladding modes can take place at the same time. This is the case in for example the sand paper test.

On the other hand the results shown in this paper illustrates that a lot of information can be drawn from a spectral microbending measurement, when a periodic microdeformation is used. In particular we get information about the propagation constants of the fiber, and the high index profile dependence of the peak positions makes measurements of these to a good characterization method for the fiber. A deformation spectrum with high spatial frequency selectivity and at the same time high reproducibility is therefore a good alternative to the former characterization method, but only if measurements are performed in a broad wavelength interval with relatively small spacings.

\section{CONCLUSION}

In this paper we have illustrated the existence of discrete cladding modes in single mode fibers. This was done by a simple spectral measurement of the additional loss due to periodically repeated microbends acting on the fiber. The characteristic peak values that appear on the spectral microbending loss curve from these measurements, is for the first time explained in detail from a theory that uses coupling between the guided mode and discrete cladding modes.

It was furthermore illustrated, that the spectral posi- tions of the loss peaks are strongly dependent on the fiber design, and detailed knowledge of the actual index profile is necessary if precise loss curves shall be predicted.

The existence of discrete cladding modes lead finally to some requirements on the choice of the artificial microbending spectrum, which is used in many fiber characterization measurements. If measurements only are performed at a few wavelengths a deformation spectrum without dominant peaks must be chosen in order to secure fair fiber comparison. Alternatively will a deformation spectrum with high spatial frequency selectivity give valuable information if a detailed spectral loss measurement is made.

\section{ACKNOWLEDGMENT}

The authors would like to thank their colleagues Dr. E. Nicolaisen, J. H. Povlsen, H. V. Pommer, and P. T. Frederiksen for fruitful discussions and helpful suggestions. Thanks are also due to LYCOM A/S for their help concerning the use of measuring equipment and for making test fibers available to us.

\section{REFERENCES}

[1] A. Tomita, P. F. Glodis, D. Kalish, and P. Kaiser, "'Characterization of the bend sensitivity of single mode fibers using the basket-weave test," in Proc. Symp. Optical Fiber Measurements, 1982, pp. 89-92.

[2] N. Kamikawa and C.-T. Chang, "Predicting microbending losses in single mode fibers," in Proc. Symp. Optical Fiber Measurements, 1986, pp. 129-132.

[3] A. O. Garg and C. K. Eoll, "New measurement technique for measurement of microbend losses in single mode fibers," in Proc. Symp. Optical Fiber Measurements, 1986, pp. 125-128.

[4] K. Petermann and R. Kühne, "Upper and lower limits for the microbending loss in arbitrary single mode fibers, "J. Lightwave Technol., vol. LT-4, no. 1, pp. 2-7, 1986.

[5] M. Artiglia, G. Coppa, and P. Di Vita, "New analysis of microbending losses in single mode fibers," Electron. Lett., vol. 22, no. 12, pp. 623-625, 1986.

[6] J. H. Povlsen and S. B. Andreasen, "Microbending theory and its implications," in Proc. 12th Eur. Conf. Opt. Commun. (Barcelona, Spain), 1986, pp. 369-372.

[7] D. Marcuse, "Microdeformation losses of single mode fibers," Appl. Opt., vol. 23, no. 7, pp. 1082-1091, 1984

[8] A. Bjarklev, "Microdeformation losses of single mode fibers with step-index profiles," J. Lightwave Technol, , vol, LT-4, no. 3, pp. $341-346,1986$.

[9] D. Marcuse, Theory of Dielectric Optical Waveguides. New York: Academic, 1974.

[10] H. G. Unger, Planar Optical Waveguides and Fibers. Oxford, England: Clarendon, 1977.

[11] P. L. Danielsen, "Analytical expressions for group delay in the far field from an optical fiber having an arbitrary index profile," IEEE J. Quantum Electron., vol. 19, pp. 850-853, 1981.

[12] P. Danielsen, "Microbending losses in single mode fibers," Electromagnetics Institute, Rep. R 273, 1983.

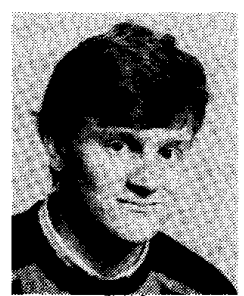

Christian B. Probst was born in Copenhagen, Denmark, on August 27, 1960. He received the M.Sc. degree in electrical engineering in 1987 from the Electromagnetics Institute, Technical University of Denmark, Lyngby.

In 1988 he joined the Copenhagen Telephone Company, Net Division, where he has been engaged in specifications of optical fiber cables. 


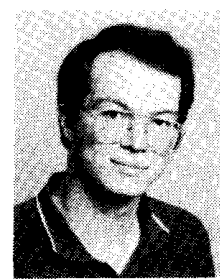

of Denmark.
Anders Bjarklev was born in Roskilde, Denmark, on July 2, 1961. He received the M.Sc. degree in electrical engineering from the Electromagnetics Institute, Technical University of Denmark, Lyngby, Denmark, in September 1985 , and the Ph.D. degree from the Technical University of Denmark in June 1988.

His research interests are primarily within the field of characterization of optical fibers. He is presently employed as Assistant Professor at the Electromagnetics Institute, Technical University

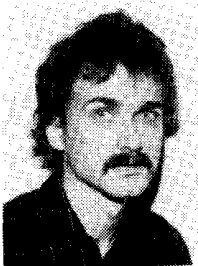

Svend Bank Andreasen was born in Vadum, Denmark, on December 12, 1959. He received the M.Sc. degree in electrical engineering in 1985 from the Electromagnetics Institute at the Technical University of Denmark, Lyngby, Denmark.

He worked as an Associate Researcher at the Electromagnetics Institute, Technical University of Denmark, where his research was in the field of characterizing and optimizing optical fibers. He is now with the R\&D Laboratory at Jutland Telephone, Tranbjerb, Denmark. 